\title{
LOS YACIMIENTOS DEL PLEISTOCENO INFERIOR DE BARRANCO LEÓN Y FUENTE NUEVA 3 DE ORCE (GRANADA). CONTRIBUCIÓN AL CONOCIMIENTO DEL PRIMER POBLAMIENTO HUMANO DE EUROPA
}

\section{THE LOWER PLEISTOCENE SITES OF BARRANCO LEÓN AND FUENTE NUEVA 3 (ORCE, GRANADA, SPAIN). CONTRIBUTION TO THE KNOWLEDGE OF THE FIRST HUMAN SETTLEMENT OF EUROPE}

\author{
por \\ ISIDRO TORO ${ }^{1}$, \\ ALAIN TURQ ${ }^{2}$, \\ JORGE AGUSTI ${ }^{3}$, \\ BIENVENIDO MARTÍNEZ-NAVARRO ${ }^{4} \mathrm{Y}$ \\ ORIOL OMS $^{5}$
}

RESUMEN En los últimos años numerosas investigaciones han contribuido a un mejor conocimiento de los primeros habitantes de Europa. Si en los años ochenta, Francia parecía situarse en el centro del debate con yacimientos como Chilhac, Soleilhac o Vallonet; los hallazgos en la región del Caúcaso y Oriente Medio (Dmanisi y Ubeidiya), la Península Itálica (Ceprano) y la Península Ibérica (Orce) en los años noventa, han desplazado el debate a las puertas de Europa. La cuestión sobre las rutas alternativas de Gibraltar o Sicilia permanece abierta. Las industrias líticas descubiertas en los yacimientos del Pleistoceno Inferior de Barranco León y Fuente Nueva 3 de Orce (Granada) se sitúan actualmente en el corazón de este debate.

\begin{abstract}
An important number of researches about the first inhabitants of Europe have been developed. If France was the centre of the debate during eighties through the sites of Chilhac, Soleilhac or Vallonet, during nineties the debate was displaced at the gates of Europe: The founds of the Caucasian region and the Middle East (Dmanisi and Ubeidiya), the Italian Peninsula (Ceprano) and the Iberian Peninsula (Orce). The questions about alternative routes, Gibraltar or Sicily, remains open. The lithic artefacts found at the Lower Pleistocene sites of Barranco León and Fuente Nueva 3 (Orce, Granada) are now in the core of this debate.
\end{abstract}

Palabras claves Pleistoceno Inferior, Industrias Líticas, Orce, Europa.

Key words Lower Pleistocene, Lithic Artefacts, Orce, Europe.

1. Delegación Provincial Consejería de Cultura, Junta de Andalucía,18008 Granada.

2. Museo Nacional de Prehistoria, 24620 Les Eyzies de Tayac. Francia.

3. Instituto de Paleontología “ Dr. M. Crusafont”, Diputación de Barcelona. 08201 Sabadell (Barcelona).

4. Orce Research Project, Venta Micena, 18805 Orce (Granada).

5. Departamento de Geología, unidad de Estratigrafía, Universidad Autónoma de Barcelona, 08193 Bellaterra (Barcelona). 


\section{INTRODUCCIÓN}

En los últimos veinte años, numerosa investigaciones han contribuido a un mejor conocimiento de los primeros habitantes de Europa. Si en los años ochenta, Francia parecía situarse en el corazón del debate con los yacimientos de Chilhac, Soleilhac o Vallonet, los años noventa han modificado radicalmente esta percepción. Los hallazgos de Dmanisi en Georgia, de Orce en España, Ceprano y Venosa en Italia y Ubeidiya y Latamé en Oriente Medio han desplazado el debate, allí donde siempre debió estar, no en el centro de Europa sino a sus puertas: el Estrecho de Gibraltar, Sicilia y el Oriente Medio.

En África, la evolución de las industrias líticas es bien conocida. La imagen del primer hombre fabricando únicamente cantos tallados tiene tendencia a esfumarse desde que se sabe que estos útiles macizos (choppers y chopping tools) corresponden mayoritariamente a núcleos y, de otra parte, a piezas ligadas a actividades determinadas con una larga perduración a lo largo de toda la prehistoria. Los primeros conjuntos de objetos tallados son de lascas, concepto flexible, correspondiente a la fracturación de un bloque de roca de materias diversas para producir fragmentos. Los filos de estas lascas pueden ser utilizados tal cual o modificados mediante el retoque. Estas características corresponden al Oldowayense o, para otros investigadores, modo 1 . No es hasta varios centenares de años después cuando aparece el Achelense o modo 2, que consiste en realizar útiles a partir de un bloque de una manera organizada y simétrica.

Al lado de este esquema africano, el descubrimiento en los yacimientos no africanos de edad más antigua de los dos tipos de industrias supone poner sobre la mesa el problema de las modalidades de dispersión. La discusión está abierta. Si los autores están de acuerdo generalmente para ver varias salidas de África, las divergencias aparecen cuando se trata de buscarles una explicación y avanzar hipótesis.

Allí donde las primeras oleadas de emigrantes parecen más antiguas (entorno a los 2 millones de años) estas han dejado trazas de industrias del modo 1 desplegándose hacia Asia y dejando huellas en Dmanisi, Tajikistán, Pakistán y Java. Allí donde las segundas, más importantes, corresponden al modo 2 (o Achelense) y que son las que quizá marquen verdaderamente la definitiva colonización de Europa, los vestigios más antiguos (anteriores al millón de años) están todos localizados al sur del Mediterráneo, en el Magreb, en la región de Casablanca, en Oriente Medio, en Israel y Siria. Estos no aparecerán en Europa hasta casi los 500.000 años, en Boxgrove en Inglaterra, Venosa en Italia y en el norte del Caúcaso y, probablemente, a lo largo de los cauces de los grandes ríos de Europa occidental: Guadalquivir, Tajo, Garona, Somme,...

¿Cuáles son las primeras evidencias en Europa? De más en más numerosas y cada vez mejor datadas, estas corresponden siempre a industrias dominantemente de lascas: Orce, Vallonet, Ceprano, Monte Poggiolo, Soleilhac, Atapuerca, Korolevo, Isernia,... Así pues, tenemos entre alrededor de 1 millón y los 500.000 años, al norte del Mediterráneo, el desarrollo de unas industrias del modo 1 y al sur del modo 2.¿Cómo explicar esta diferencia? Las explicaciones son múltiples, para ciertos autores las trazas de ocupación más antiguas de Europa corresponden al retorno hacia el oeste de los primeros ocupantes de Asia. Para otros, antes de aceptar este cuadro es necesario esperar a tener de cada uno de los sitios series líticas más abundantes para estar seguros que estas industrias de lascas no son relacionables con el Achelense.

Tal como nosotros lo vemos, las industrias descubiertas en Barranco León y Fuente Nueva 3 se sitúan en el corazón de este debate en tanto que estas son, por ahora, según las últimas dataciones publicadas y contrastadas de paleomagnetismo, las más antiguas de Europa occidental. Las poco más de dos centenares de piezas descubiertas en estratigrafía en estos yacimientos de Orce son todas muy parecidas. Están todas producidas con percutor duro; las lascas obtenidas han sido utilizadas, tal cual, para cortar o rascar; la forma de los núcleos demuestra que los métodos utilizados son los característicos del modo 1 y, además, la ausencia total de la menor traza de piezas bifaciales y la no utilización del percutor blando, las separan claramente de las industrias achelenses o de modo 2 . 


\section{LA CUENCA DE GUADIX-BAZA}

\subsection{El marco geográfico}

Localizada al noreste de la provincia de Granada, la cuenca de Guadix-Baza está limitada al norte por la Sierra de Segura y la Sierra de la Sagra y al sur y al este por una serie de altos relieves que la cierran en parte, Sierra Nevada, Sierra de Baza, Sierra de las Estancias, Sierra de Orce y Sierra de María. El sector de Orce, situado en la parte oriental de la cuenca se presenta actualmente como un altiplano $(900 \mathrm{~m})$ bordeado al norte y al sur por montañas calcáreas que culminan entre 1.300 y $2.380 \mathrm{~m}$. Ampliamente abierta hacia el oeste sobre el conjunto de la depresión, está limitada al este por el paso de María, pasaje hacia tierras murcianas, y al noreste por una falla dominada por relieves formados por conglomerados de cantos. En mitad de los depósitos palustres y lacustres plio-pleistocenos, se eleva un relieve importante de $1.100 \mathrm{~m}$, el cerro de la Venta. Dos valles subparalelos fuertemente encajados, la Cañada de Vélez y la Cañada del Salar, dan acceso a cortes de más de 60 metros de potencia, en los que afloran los yacimientos arqueológicos y paleontológicos que nos permiten reconstruir la historia paleobiológica de los dos últimos millones de años.

\subsection{El marco geológico}

La geología de la cuenca de Guadix-Baza es ampliamente conocida gracias a los numerosos trabajos estratigráficos realizados (Vera 1969; 1970 a y b y Vera et al. 1985). Es una cuenca postorogénica intramontañosa, originada en la fase distensiva que siguió a la formación de las Cadenas Béticas. En ella se distinguen dos subcuencas que corresponden a la subcuenca de Guadix (en el sector occidental) con sedimentos fluviotorrenciales, esencialmente aluviales, y al subcuenca de Baza (en el sector oriental) de composición más variada que corresponde a la parte lacustre del paleolago (Fig.1). Entre ambas se eleva la Sierra de Baza y más al norte el umbral del Jabalcón, ambos formados a partir de materiales de las Zonas Internas Béticas.

Los materiales neógeno-cuaternarios que rellenan la cuenca reposan discordantemente sobre materiales plegados y erosionados, tanto de las Zonas Internas como de las Zonas Externas Béticas. El contacto entre ambas zonas queda cubierto por dicho relleno sedimentario en una longitud de unos $80 \mathrm{~km}$.

Los materiales más modernos de la cuenca corresponden a un nivel geomorfológico (glacis) y sedimentario (conglomerados) sin apenas deformación posterior llamado usualmente "nivel de colmatación" (Vera1970) y que actualmente se sitúa a unos mil metros de altitud. La red fluvial actual está encajada en los materiales subhorizontales más modernos, habiendo producido un rápido vaciado parcial de los mismos (Calvache y Viseras 1997). Algunos de los ríos actuales (Guadiana Menor, Fardes, Gor, etc.) se han encajado de manera notable dando lugar a gargantas y cañones de paredes subverticales que ofrecen excelentes panorámicas de los materiales del relleno sedimentario.

\subsection{El marco cronobioestratigráfico}

Basada fundamentalmente en los estudios sobre los micromamíferos (Agustí et al. 1987; Agustí 1996; Oms et al. 1999), además de los de macromamíferos (Martínez Navarro 1991; Martínez Navarro et al. 2000) y en los datos paleomagnéticos (Oms et al. 1994,1996 y 2000; Agustí et al. 1997 y 1999), se ha podido establecer una biozonación fiable que abarca desde el Mioceno hasta el Pleistoceno Superior. Tres biozonas han sido reconocidas, de abajo a arriba, para el Pleistoceno Inferior: Biozona con Allophaiomys pliocaenicus, bien representada en el yacimiento de Venta Micena de Orce; biozona con Allophaiomys burgondiae, presente 
en Barranco León y Fuente Nueva 3 de Orce, Loma Quemada, Huéscar 1 y Puerto Lobo de Huéscar y, biozona con Terrícola arvalidens representada en Cúllar-Baza C; el comienzo del Pleistoceno Medio caracterizado por la biozona con Arvicola cantiana está presente en Cúllar-Baza1 y Solana del Zamborino (Agustí et al. 2000).Los yacimientos de Barranco León y Fuente Nueva 3, emplazados en la biozona con Allophaiomys burgondiae, poseen, en base a los datos paleomagnéticos, una edad anterior a Jaramillo (Oms et al. 2000), habiendo sido encontradas en ellos las primeras evidencias de una temprana ocupación humana de la cuenca y de Europa occidental.

\section{LOS YACIMIENTOS DE BARRANCO LEÓN Y FUENTE NUEVA 3 DE ORCE}

\subsection{Barranco León}

Situado a unos tres kilómetros de Orce en dirección María, se trata de un barranco orientado norte-sur que nace a los pies de la Sierra de la Umbría. Excavado en 1995 por J. Gibert (Gibert et al. 1998), con posterioridad se han realizado dos campañas más de excavación en el mismo 1999 y 2000, bajo la dirección de G. Martínez Fernández e I. Toro en el marco del Proyecto de Investigación "Geología, Paleontología, Paleoecología y Arqueología de la Cuenca de Guadix-Baza durante el Plio-pleistoceno". Dichas excavaciones han permitido verificar algunas de las hipótesis anteriores y aportar algunas precisiones importantes. Se ha confirmado la existencia de al menos dos capas arqueológicas (Barranco León 1 y 5, BL1 y 5) identificadas anteriormente; la unidad estratigráfica BL-1 está constituida por un depósito en aguas poco profundas y anaeróbicas y el escaso material arqueológico encontrado se compone de restos óseos en mal estado de conservación.

La unidad estratigráfica BL-5 contiene en todo su espesor restos de fauna y material lítico tallado. Esta unidad que presenta una morfología y una potencia irregular reposa sobre una superficie erosiva que a veces afecta a la unidad estratigráfica subyacente. La hipótesis actual es que esta paleosuperficie, situada en un área marginal de la zona lacustre, ha sido fuertemente perturbada por la acción de megaherbivoros. Así mismo parece ser que fuerzas hidráulicas han podido contribuir, en cierta medida, a la constitución del depósito.

Emplazado en la biozona caracterizada por el Allophaiomys burgondiae, la macrofauna de este yacimiento se compone de Hippopotamus antiquus, Equs altidens, Felidae cf. Homoterium sp., Megaloceros sp. y Bovini gen. indet.(Gibert et al. 1998).

La industria recogida en estas campañas de excavación (centenar y medio de piezas) ha sido realizada mayoritariamente en sílex de origen local recogido en posición secundaria, cuyos afloramientos se localizan en la cercana Sierra de Orce. Las piezas son a menudo de pequeñas dimensiones, algo más de un cuarto de ellas tienen menos de $2 \mathrm{~cm}$; casi un tercio de las mismas presentan trazas de transporte por el agua, estando normalmente lustradas pero, curiosamente, con escasos signos de rodamiento. El objetivo de la talla ha sido fundamentalmente la producción de lascas (Fig. 2, no 3,4,5,6,7 y 8) extraídas según una dirección dominante que produce a veces soportes con tendencia laminar y raramente centrípeta. El importante número de fracturas diametrales (de tipo Siret) es característico de la utilización del percutor duro. Los talones son sobretodo corticales, lisos inclinados y escasos facetados. Los escasos núcleos encontrados no presentan una preparación especial utilizándose como plano de percusión la superficie natural o el negativo de una gran lasca. Un núcleo muestra dos planos de percusión independientes, dispuestos ortogonalmente, a partir de los cuales se han realizado una serie de levantamientos que se recortan. Existen algunos núcleos sobre lasca que dan productos Kombewa (Fig. $2 \mathrm{n}^{\circ}$ 2). Se identifican también algunas piezas reflejadas.

Un 14\% de los productos de talla presentan sobre un borde cortante retoque simple, discontinuo y alterno que se puede situar opuesto a un dorso natural (como los cuchillos de dorso natural), o sobre una angulación 
del soporte dando una piezas de una morfología más repetitiva que recuerdan a los bec o perforadores de escasa modificación secundaria. A falta de experimentación con materiales idénticos y de la realización de un estudio traceológico, no rehusamos su interpretación como retoques espontáneos (¿retoques de uso?) o ligados a fenómenos naturales(transporte, presión del sedimento). Si consideráramos estas modificaciones secundarias como retoques secundarios, desde el punto de vista tipológico tendríamos cuchillos de dorso naturales, muescas y denticulados.

\subsection{Fuente Nueva 3}

Este yacimiento se localiza en el anejo de Fuente Nueva, cerca de la iglesia en ruinas y de las antiguas fuentes que dan nombre al lugar, sobre una de las vertientes que dominan la cabeza del valle fluvial de la Cañada de Vélez. Descubierto en 1992 con motivo de las obras de instalación de un poste de la electricidad, en este yacimiento se han realizado varias campañas de excavación; en 1994 se realizó un sondeo y limpieza de toda la superficie afectada por las obras bajo la dirección de B. Martínez Navarro y A. Turq. En 1995 estos mismos investigadores realizan una campaña de excavación (Turq et al.1996 y Martínez Navarro et al. 1997) en codirección con J. Gibert. En 1999 y 2000 se realizan sendas campañas enmarcadas en el proyecto de investigación en curso, bajo la dirección de G. Martínez e I. Toro, las cuales han demostrado que la conservación del sitio no es uniforme presentando una estructura sedimentaria más compleja que la publicada en su momento (Martínez Navarro et al. 1997).

Localizado también en la biozona de Allophaiomys burgondiae de la bioestratigrafía regional, el yacimiento presenta la siguiente asociación faunística: Mammuthus meridionalis, Hippopotamus antiquus, Stephanorinus etruscus, Equus altidens, Megaloceros solilhacus, Cervus sp., Bovini gen. et sp. indet., Hemitragus sp., Megantereon whitei, Ursus sp., Hystrix major, Allophaiomys burgondiae, Allophaiomys chalinei, Mimomys savini y Mimomys oswaldoreigi (Martínez Navarro et al. 1997).

Poco más de una centena de piezas provenientes de sector este del yacimiento nos muestran las siguientes características: la materia prima mayoritariamente utilizada es el sílex de origen local (próximo al ópalo que se presenta en forma de cantos aluviales), la cuarzita y algunos cantos y pequeños bloques de caliza micrítica.

Desde el punto de vista tecnológico, parece que la totalidad de la cadena operatoria está representada. Son numerosas las pequeñas lascas y débris (Fig. $3 \mathrm{n}^{\circ} 7$ y 8).La técnica de talla es exclusivamente con percutor duro como lo demuestran los numerosos dobles bulbos y los accidentes tipo Siret. Los talones son normalmente corticales o lisos inclinados, muy raros los facetados aunque hay algún facetado convexo. El objetivo de la talla es la producción de lascas (Fig. $3 \mathrm{n}^{\circ}$ 6,7 y 8) directamente a partir de un bloque con cambios de orientación o sobre la cara inferior de grandes lascas (técnica Kombewa). Algunos soportes con tendencia laminar (Fig. $3 \mathrm{n}^{\circ} 1$ ) podrían ser considerados como evolucionados. Los núcleos están bien representados (Fig. $3 \mathrm{n}^{\circ} 2$ y 3 ) y varios presentan dos planos de percusión utilizados sucesivamente, las series de levantamientos son cortas y los últimos negativos se presentas a veces reflejados (Fig. $3 \mathrm{n}^{\circ}$ 5). También hay algunos productos de mantenimiento o de reutilización en forma de núcleos (Fig. $3 \mathrm{n}^{\circ} 4$ ). El utillaje se compone fundamentalmente de piezas astilladas (Fig. $3 \mathrm{n}^{\circ}$ ) así como de bec o perforadores de débil modificación secundaria (Fig. $3 \mathrm{n}^{\circ}$ 11); el único útil retocado claramente identificable es una raedera que también ha sido utilizada como pieza astillada (Fig. $3 \mathrm{n}^{\circ} 10$ ). 


\section{COMPARACIONES Y CONCLUSIONES}

Del registro bioestratigráfico y los datos del paleomagnetismo de Barranco León y Fuente Nueva 3 se pueden obtener una serie de conclusiones: La presencia de una magnetización inversa a lo largo de su estratigrafía, combinada a las asociaciones faunísticas que presentan, indican una edad Matuyama (0,78-1.77 Ma) para estos yacimientos arqueológicos del Pleistoceno Inferior. La presencia de M. nivaloides en Le Vallonet en sedimentos con polaridad normal adscritos al subcron Jaramillo (0.99-1.07 Ma), una especie más joven que el Allophaiomys burgondiae de Barranco León y Fuente Nueva 3 indica que la fauna y las industrias líticas de ambos yacimientos pertenecen al cron inverso Matuyama y por lo tanto son más antiguas de 1.07 Ma (Oms et al. 2000).

En general, el aspecto de las industrias de lascas de Barranco León y Fuente Nueva 3 se aproxima mucho al de las industrias arcaicas de sitios como Dmanissi en Georgia (en las capas II y III los útiles con muescas -denticulados, astillados, bec...-ascienden al 70\%), o a las de el Lacio italiano, Colle Marino, Arce, Fontana Liri, Ceprano, Le Vallonet en Francia, o Atapuerca en España; se trata de una talla rápida y oportunista, caracterizada por un golpeo muy violento con percutor duro, lo que confirma la abundante presencia en ellas de accidentes de talla tipo Siret y dobles bulbos. El objetivo principal consistía en producir rápidamente, sin procedimientos tecnológicos muy avanzados, un importante número de lascas de filos cortantes que eran empleadas directamente, sin modificar con el retoque, para realizar diversa actividades.

La casi ausencia en Barranco León y Fuente Nueva 3 de cantos tallados, o la existencia de soportes con tendencia laminar o centrípeta, que las diferencia de las anteriores y les da un aspecto como más evolucionado (Nioradzé et al. 2000), es un dato que debe tomarse con las debidas reservas derivadas de lo exiguo de la superficie excavada en ambos yacimientos y de las muestras líticas sobre las que se trabaja, debiendose esperar a la realización de nuevas campañas de excavación y nuevos estudios sobre una muestra suficientemente amplia de materiales líticos para fijar posiciones en dicho aspecto.

Como señalábamos en la introducción a este artículo, los yacimientos de Barranco León y Fuente Nueva 3 de Orce se sitúan en el corazón de debate sobre el primer poblamiento humano de Europa, en tanto que demuestran la presencia humana en Andalucía hace más de un millón de años, configurándose pues como sitios clave para la identificación de los grupos humanos que ocuparon el área mediterránea durante la fase cronológica que precedió a la difusión de las industrias con bifaces más antiguas.

\section{BIBLIOGRAFÍA}

AGUSTÍ, J. (1986): "Synthèse biostratigraphique du Plio-pleistocene de Guadix-Baza (Province de Granada, Sud-Est de L'Espagne)", Geobios 19(4): 505-510.

AGUSTÍ, J.; MOYÂ-SOLÁ, S. y PONS-MOYÁ, J. (1987): “La sucesión de mamíferos en el Pleistoceno inferior de Europa: proposición de una nueva escala bioestratigráfica", Paleontología y Evolución, Memoria Especial 1: 287-295.

AGUSTÍ, J.; OMS, O.; GARCÉS, M. y PARÉS, J. M. (1996): "Calibration of the late Pliocene- early Pleistocene transition in the continental beds of the Guadix-Baza Basin (South-Eastern Spain)", Quaternary International 40: 93-100.

AGUSTÍ, J.; OMS, O.; y PARÉS, J. M.(1999): "Calibration of the early- Middle Pleistocene transition in the continental beds of the Guadix-Baza Basin (SE Spain)", Quaternary Science Revue 18: 1409-1417. 
AGUSTÍ, J.; MARTÍNEZNAVARRO, B.; OMS, O. y TORO, I.(2000): “The Plio-pleistocene mammalian succession from the Guadix-Baza Basin (Andalousia, Spain). Biostratigraphical and paleoencironmental background", Resumes des communications, Colloque sur "les Premiers Habitants de l'Europe", Avril 2000, Tautavel.

CALVACHE, J. L.y VISERAS, C.(1997): "Long term control mechanism of stream piracy processes in southeast Spain", Earth Surf. Process. Landform 22: 93-105.

GIBERT, J.; IGLESIAS, A.; MAÍLLO, A. y GIBERT, L.(1998): “ Two Oldowan assemblagesin the Pliopleistocene deposits of the Orce región, southeast Spain", Antiquity 72: 17-25.

MARTÍNEZ NAVARRO, B.(1991): Revisión sistemática y estudio cuantitativo de la fauna de macromamíferos del yacimiento de Venta Micena (Orce, Granada). Tesis doctoral Universidad Autónoma de Barcelona, edición en microfilm: 264, lám. 34, Bellaterra.

MARTÍNEZ NAVARRO, B.; TURQ, A.; AGUSTÍ, J. y OMS, O. (1997): "Fuente Nueva 3 (Orce, Granada, Spain) and the first human occupation of Europe", Journal of Human Evolution 33: 611-620.

MARTÍNEZNAVARRO, B.; AGUSTÍ, J.; OMS, O. y TORO, I. (2000): "The continental Plio-pleistocene series of the Guadix-Baza Basin (South-eastern Spain)”, Abstracts of the SEQS 2000 Meeting “The Plio-pleistocene boundary and the Lower-Middle Peistocene transition: Type areas and sections". Bari.

NIORADZE, M.; DE LUMLEY, H.; BARSKY, D.; CAUCHE, D.; CELIBERTI, V.; NOTTER, O.; BIDITTU, I.; KILADZE, G. et ZVANIA, D. (2000): "Les indiustries lithiques archaiques du site de Dmanissi, Georgie. Comparaisons avec les indutries archaiques de l'Afrique de 1'Est et de 1'Europe méridionale", Resumés des communications, Colloque International sur "les Premiers Habitants de 1'Europe" Avril 2000. Tautavel.

OMS, O.; GARCÉS, M.; PARÉS, J. M.; AGUSTÍ, J.; ANADÓN, P. y JULIÁ, R. (1994): “Magnetostratigraphic characterización of a tick lower Pleistocene lacustrine sequence from the Baza Basin (Betic chain, Southern Spain)", Phys. Earth Planet International 85.

OMS, O.; AGUSTÍ, J.; GABÁS, M. y ANADÓN, P. (1999): "Lithostratigrphical correlation of the micromammalian sites and biostratigaphy of the Upper Pliocene to Lower Pleistocene in the Northeast Guadix-Baza Basin(Southern Spain)", Journal Quaternary Science 15: 43-50.

OMS, O.; PARÉS, J. M.; MARTÍNEZ NAVARRO, B.; AGUSTÍ, J.; TORO, I.; MARTÍNEZFERNÁNDEZ, G. y TURQ, A. (2000): "Early human occupation of Western Europe: Paleomagnetic dates of two peleolithic sites in Spain", Proceeding of the National Academy of Sciences 97 (19): 10666-10670.

TURQ, A.; MARTÍNEZ NAVARRO, B.; PALMQVIST, P.; ARRIBAS, A.; AGUSTÍ, J. y RODRÍGUEZ VIDAL, J. (1996): "Le Plio-pleistocene de la région d'Orce, province de Grenade, Espagne: Bilan et perspectives de recherche", Paleo 8: 161-204.

VERA, J.A. (1969): "Características estratigráficas de la serie de Baza”, Depresión de Guadix-Baza”, Acta Geológica Hispana 4: 14-17.

VERA, J. A. (1970 a ): "Estudio estratigráfico de la Depresión de Guadix-Baza", Boletín Geológico y Minero 81: 429-462.

VERA, J. A. (1970 b): "Facies del Plioceno de la Depresión de Guadix-Baza", Cuadernos de Geología Universidad de Granada 1: 23-25.

VERA, J. A.; FERNÁNDEZ, J.; LÓPEZ-GARRIDO, A. C. y RODRÍGUEZ FERNÁNDEZ, J.(1985): "Geología y Estratigrafía de los materiales plioceno-pleistocenos del sector Orce-Venta Micena (Prov. de Granada). Paleontologia y Evolució, 18: 3-11. 


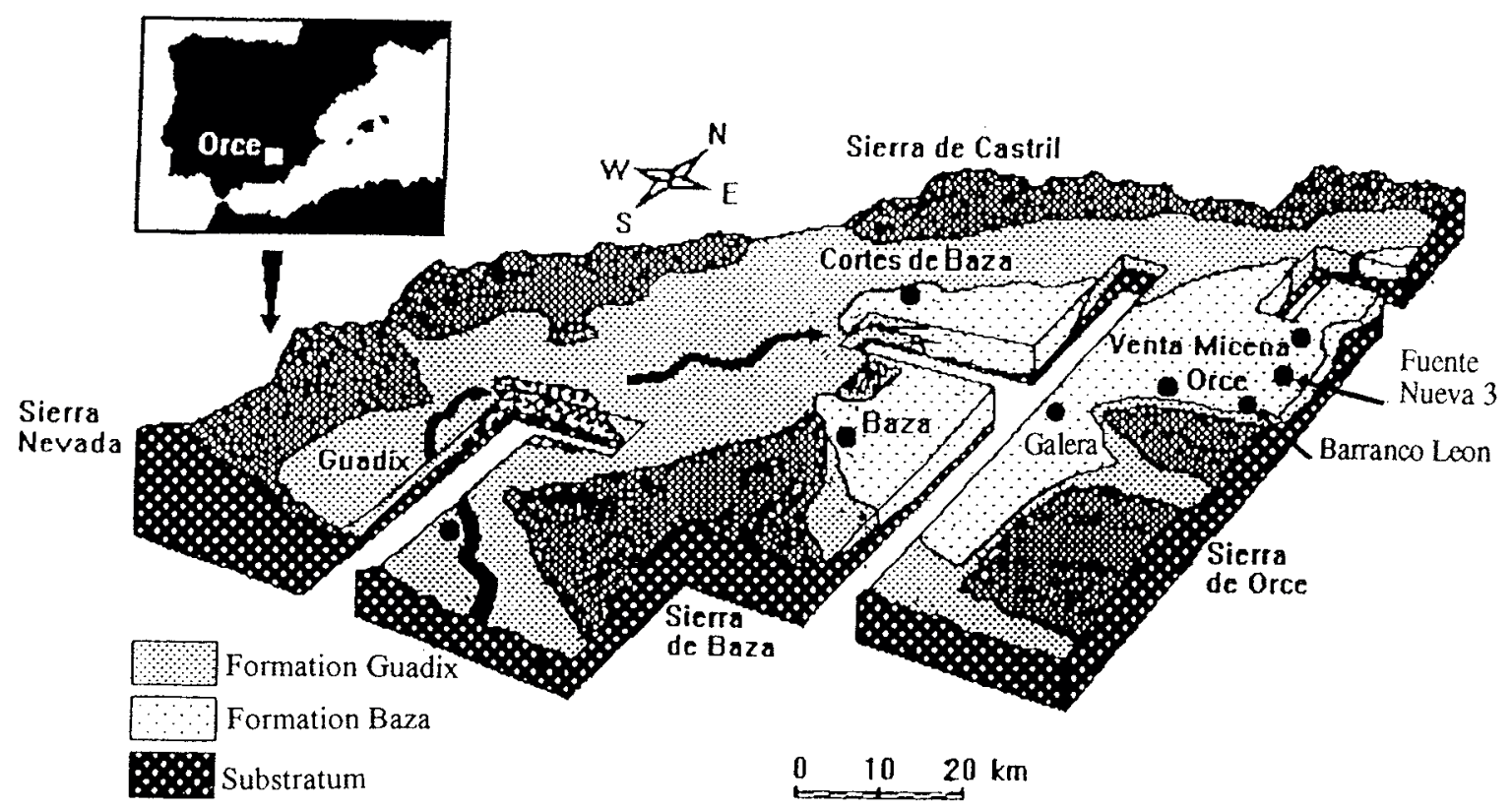

Figura 1. Bloque diagrama de la Cuenca de Guadix-Baza. Situación de los principales yacimientos. 


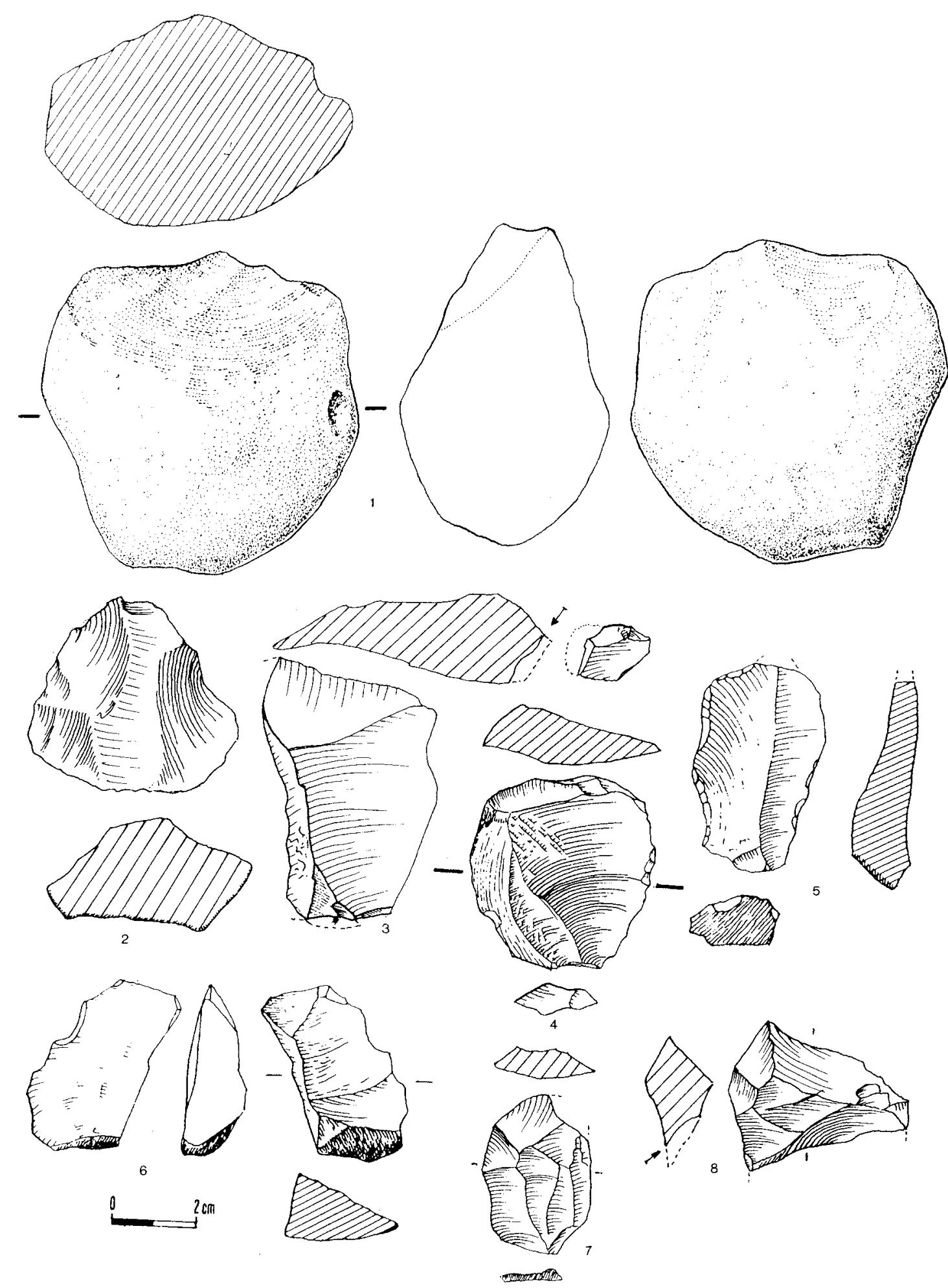

Figura 2. Industria lítica de Barranco León. (Dibujo A. Turq).

ISSN: 1133-4525 ISSN-e: 2255-3924

SPAL 9 (2000)

http://dx.doi.org/10.12795/spal.2000.i9.09 

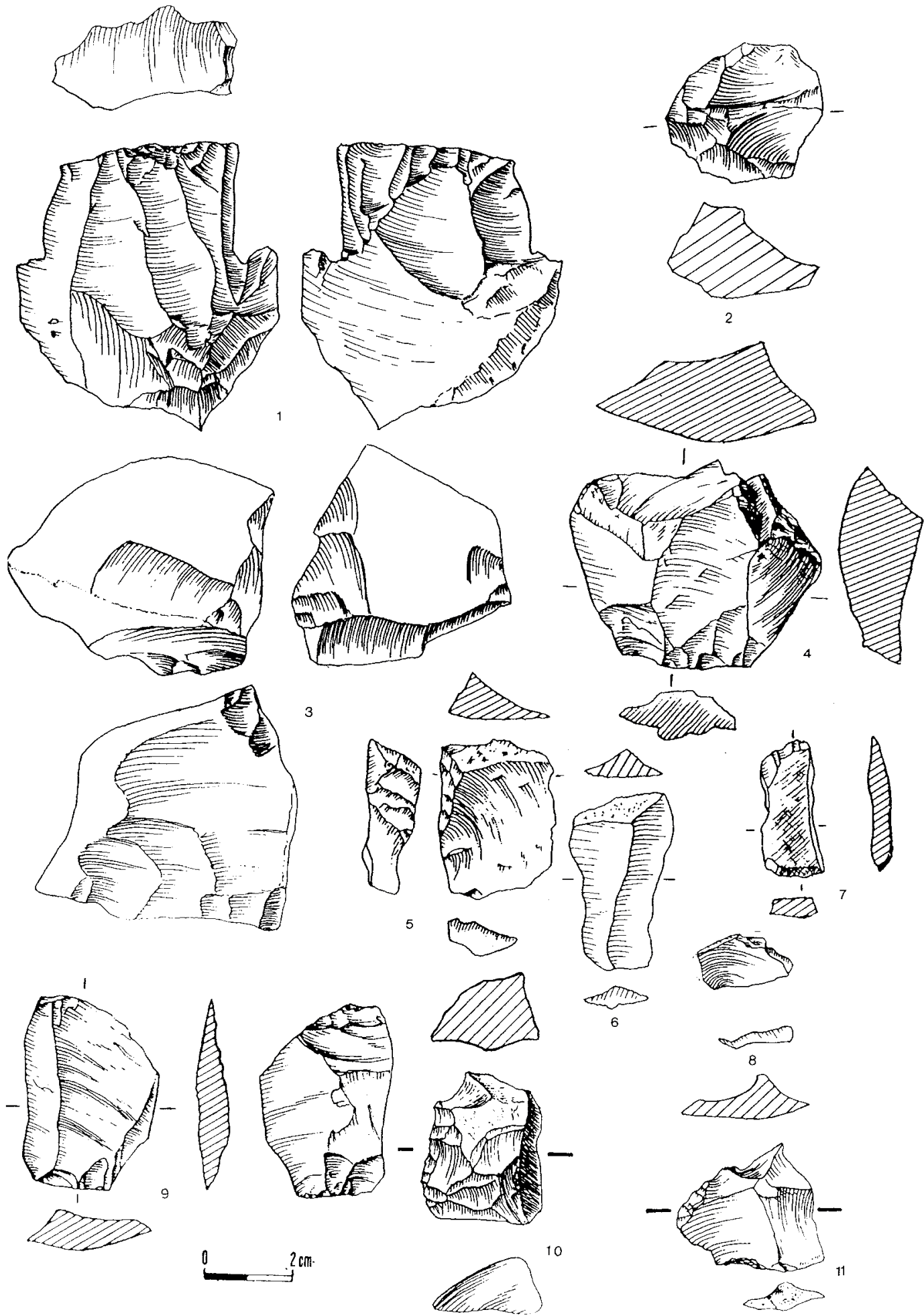

$\underbrace{m}_{6}$
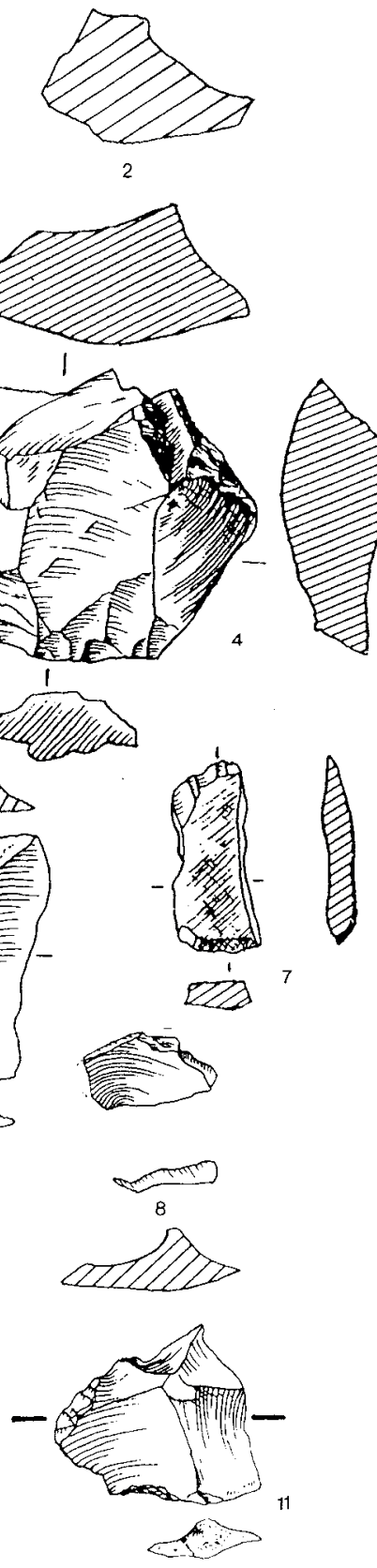

Figura 3. Industrias líticas de Fuente Nueva 3. (Dibujo A. Turq). 